Recent Advances in Communication, Electronics \& Electrical Engineering

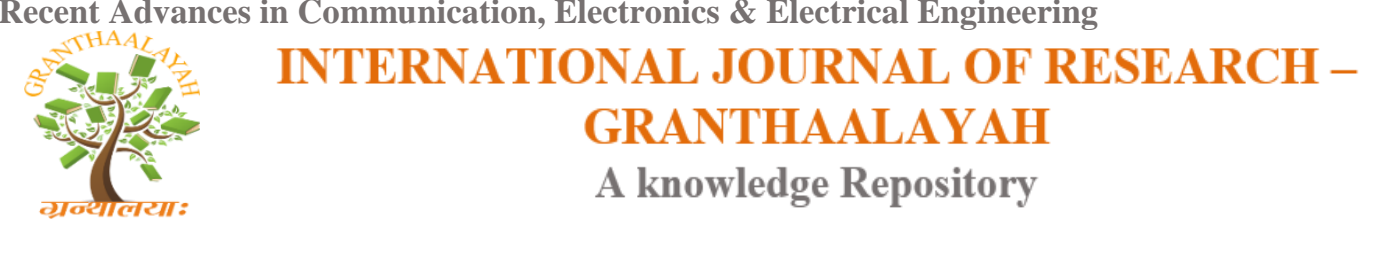

RACEEE - 17

\title{
IMAGE DENOISING USING WAVELET AND SHEARLET TRANSFORM
}

\author{
Bharath Kumar $S^{1}$, Kavyashree $S^{2}$, Ananth V Naik ${ }^{3}$, Kavyashree C.L ${ }^{4}$, Gayathri K.M ${ }^{5}$ \\ 1,2,3,4 UG Students, Department of Electronics and Communication Engineering, School of \\ Engineering and Technology, Jain University, Bangalore-562112, India \\ ${ }^{5}$ Assistant Professor, Department of Electronics and Communication Engineering, School of \\ Engineering and Technology, Jain University, Bangalore-562112, India
}

DOI: https://doi.org/10.29121/granthaalayah.v5.i4RACEEE.2017.3316

\begin{abstract}
Image plays an important role in this present technological world which further leads to progress in multimedia communication, various research field related to image processing, etc. The images are corrupted due to various noises which occur in nature and poor performance of electronic devices. The various types of noise patterns observed in the image are Gaussian, salt and pepper, speckle etc. due to which the image is attenuated or amplified. The main challenge lies in removing these noises. We use various denoising techniques in removal of noise in order to retrieve the original information from the image. Wavelet transforms are one of the denoising algorithms used as conventional methods. This algorithm is used to capture the image along different directions in limited manner which becomes the main disadvantage of using this algorithm. In this work we propose a technique by integrating Wavelet and Shearlet transform which effectively removes the noise to the maximum extent and restores the image by edge detection which can be identified. The simulation is done on synthetic image and shows improvement with existing methods. The algorithm is simulated in MATLAB 2016b.
\end{abstract}

Keywords: Types of Noise; Denoising Filters; Histogram; Wavelet Transform; Shearlet Transform; MATLAB 2016b.

Cite This Article: Bharath Kumar S, Kavyashree S, Ananth V Naik, Kavyashree C.L, and Gayathri K.M. (2017). "IMAGE DENOISING USING WAVELET AND SHEARLET TRANSFORM." International Journal of Research - Granthaalayah, 5(4) RACEEE, 8-14. https://doi.org/10.29121/granthaalayah.v5.i4RACEEE.2017.3316.

\section{Introduction}

Image restoration is very significant field of image processing so, image denoising is necessary. It is a process used for removing noise and enhance the quality of the image by preserving the 
Recent Advances in Communication, Electronics \& Electrical Engineering

original information. Designing a denoising method is a challenging task. We need to know the various noise patterns in order to implement the suitable algorithms.

\subsection{Types of Noises}

- Gaussian noise: It occurs while capturing an image, due to sensor noise caused by dim light or high temperature. Its distribution is even throughout the signal. This means that each pixel in the noisy image is the sum of the true pixel value and a random Gaussian distributed noise value. Gaussian noise is also known as white noise. Here the samples are sequential in time or arranged along one or more spatial dimensions. The pixels of a white noise are typically arranged in a rectangular grid in digital image processing and assumed to be independent random variable with uniform probability distribution over some interval. Usually the noises are represented using histogram.

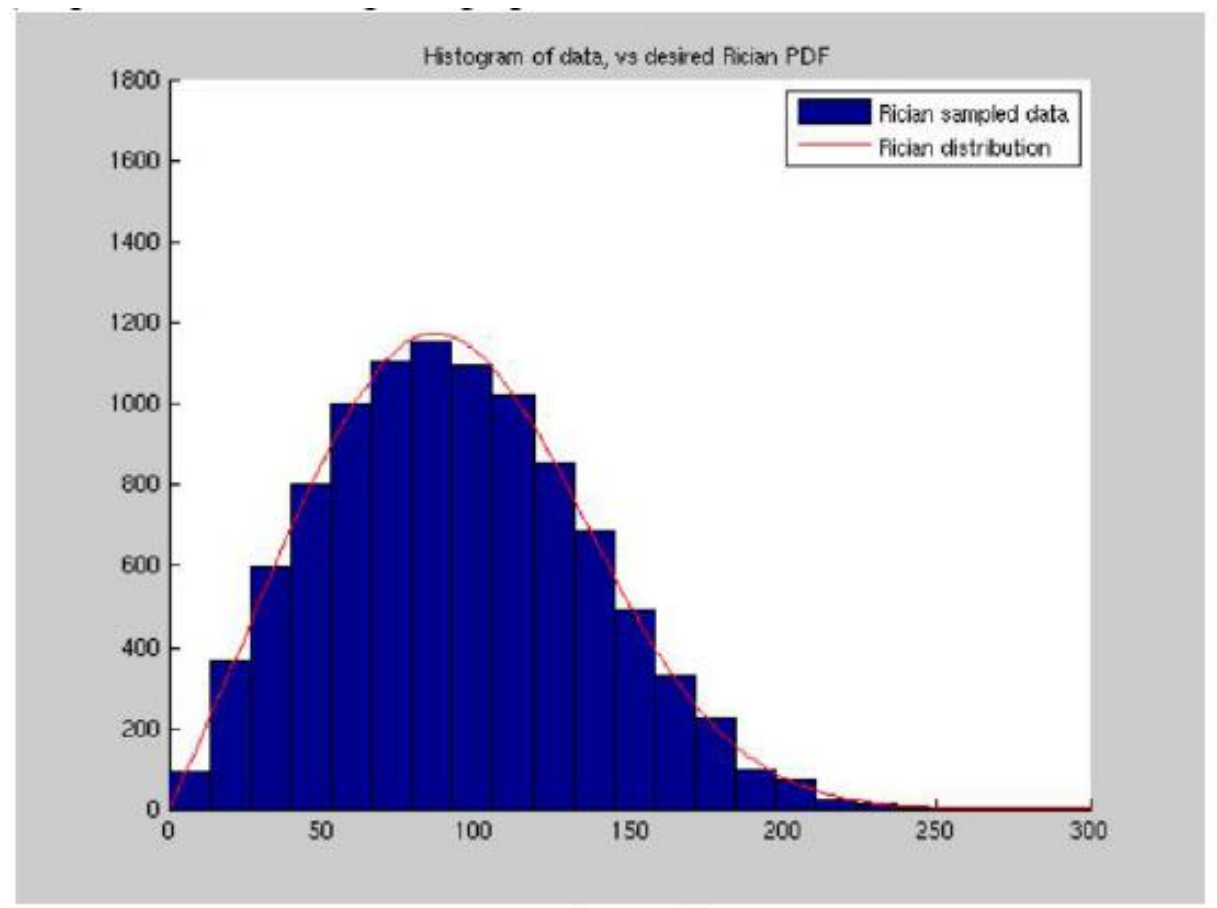

Figure 1:

The figure (1) represents the histogram graph of Gaussian noise.

- Salt and pepper: Salt and pepper noise consists of dark pixels in bright regions and bright pixels in dark regions. salt and pepper noise is introduced in an image by setting a fraction of $r / 2$ for randomly selected pixels to black, and another fraction of $r / 2$ randomly selected pixels to white. It is also impulsive noise or thermal noise. Denoising this noise is done by low pass filters. A-D convertor errors, bit errors in transmission usually create this type of noise. Figure (2) represents the histogram graph of salt and pepper noise. 
Recent Advances in Communication, Electronics \& Electrical Engineering

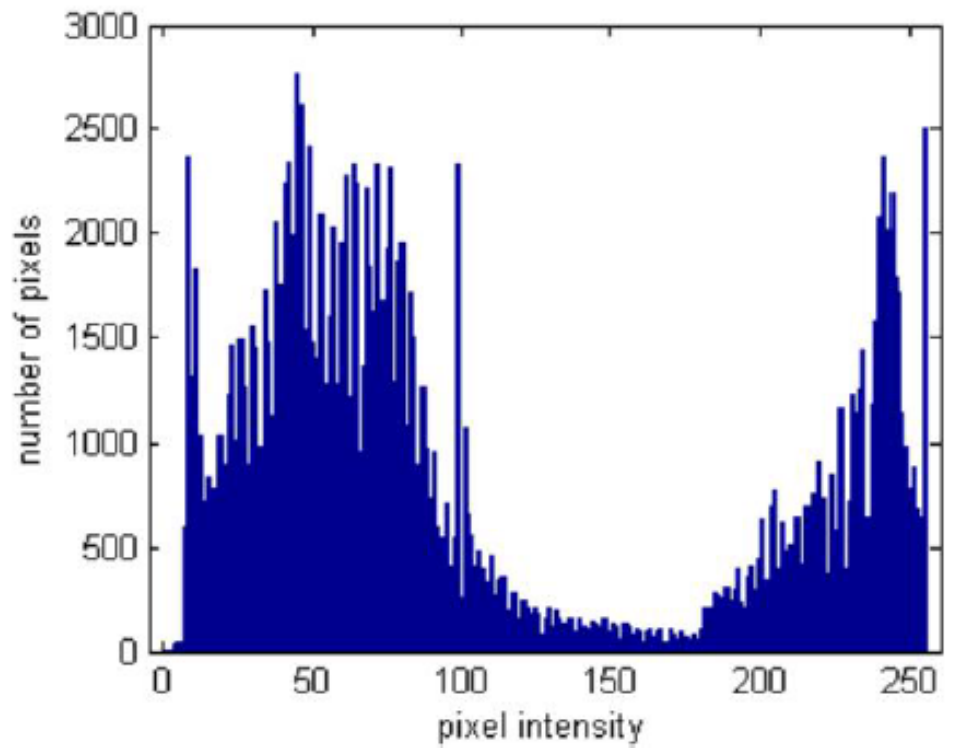

Figure 2:

- Speckle noise: Speckle noise is a granular noise which degrades the quality of the active radar, synthetic aperture radar (SAR), medical ultrasound images due inherent existence. Speckle noise appears due to random fluctuations in the return signal from an object which is in the size of single image processing element. It also increases the mean gray level of a local area. It consists of some important information, especially when it is linked to the laser speckle and to the dynamic speckle phenomenon. Speckle noise is also called as impulsive noise. This noise is found more in satellite images. Figure (3) represents the histogram graph of speckle noise.

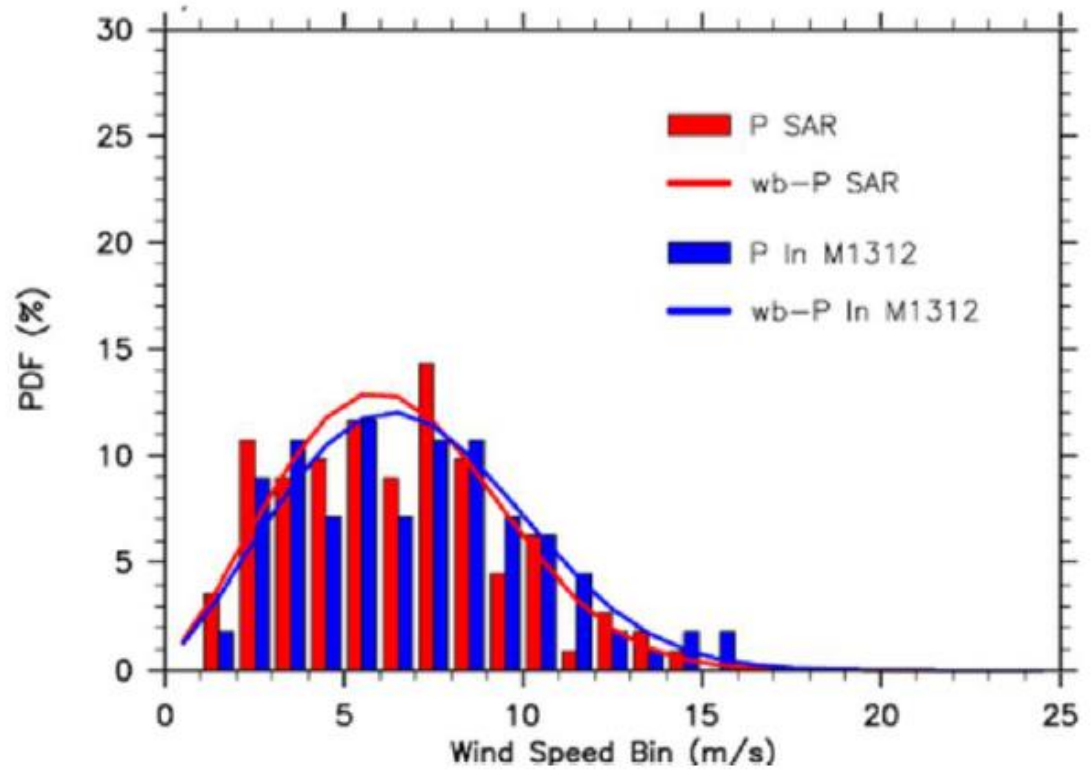

Figure 3: 
The other denoising technique called as wavelet transforms which are the most conventional method.

- Wavelet transforms: The term wavelet is referred to as wave like oscillation whose amplitude begins at zero, increases, and then decreases back to zero. These are mathematical functions that analyze data according to scale or resolution. Generally Wavelet Transforms are of two types namely Continuous and Discrete Wavelet Transforms.

Consider original signal $f(x)$, then the continuous wavelet transform of the signal $f(x)$ is defined by

$$
W_{\varphi}(s, \tau)=\int_{-\infty}^{\infty} f(x) \Psi_{s, \tau}(x) d x
$$

This transforms a continuous function of one variable into a continuous function of two variables i.e. translation and scale. The wavelet coefficients measure how closely correlated the wavelet is with each section of the signal. We choose a wavelet that matches the shape of the image components for compact representation like in Haar wavelet for black and white drawings.

Unlike continuous wavelet transforms there is no necessity to calculate wavelet at every possible scale in discrete wavelet transforms. We can choose scales based on powers of two, and get equivalent accuracy.

$$
\Psi_{j, k}(x)=2^{j / 2} \Psi\left(2^{j} x-k\right)
$$

Discrete function can be represented $f(n)$ as weighted summation of wavelets $\psi(n)$ and coarse approximation $\varphi(n)$.

$$
f(n)=\frac{1}{\sqrt{M}} \sum_{k} W_{\varphi}\left(j_{0}, k\right) \varphi_{j_{0}, k}(n)+\frac{1}{\sqrt{M}} \sum_{j=j_{0}}^{\infty} \sum_{k} W_{\varphi}(j, k) \Psi_{j, k}(n)
$$

They approximate signals with sharp spikes or signals having discontinuities and also they model speech, music, video and non-stationary stochastic signals. Wavelets provide some advantage over Fourier transforms. In Fourier transforms, signals are represented as a sum of sinusoids. Wavelets transforms the wavelets are localized in both time and frequency unlike in Fourier transform where signals are localized only in frequency. The Short-time Fourier transform is similar to the wavelet transform but there is a trade-off between frequency and resolution. They have wide applications such as image compression, removing of noise effectively, turbulence, human vision, radar, earthquake prediction, etc. 
Recent Advances in Communication, Electronics \& Electrical Engineering

- Shearlet transforms: Shearlet transforms are very useful in capturing and preserving the intrinsic properties of a multidimensional image. It is useful in de-noising the multidimensional images hence it is called multiscale transform. There are two types in Shearlet transforms namely continuous and discrete Shearlet transform. Shearlet can also be used to analyze multivariate and multidimensional data.

Shearlet transform is represented by the mathematical equation $\|f-f n\|_{L^{2}}^{2} \leq C N^{-2}(\log N)^{3}, N \rightarrow \infty$,

Where constant $C$ depends on the maximum curvature of the singularity curve and maximum magnitudes of $f$. $N$ is the largest Shearlet coefficient, as $N$ increases the error decreases. $f$ is the sparse approximation function. $f n$ is nonlinear Shearlet representation of signal $f$ with $N$ largest Shearlet coefficients.

Shearlet transforms are the improved version of wavelets but provide better results compared to wavelets and are efficient in preserving edges of an image while de-noising but in the case of wavelet transform the image edges will be deteriorated. Shearlet are efficient transforms for edge analysis and detection. In order to remove and enhance image we use both wavelet and Shearlet transforms in this paper.

\section{Proposed Block Diagram}

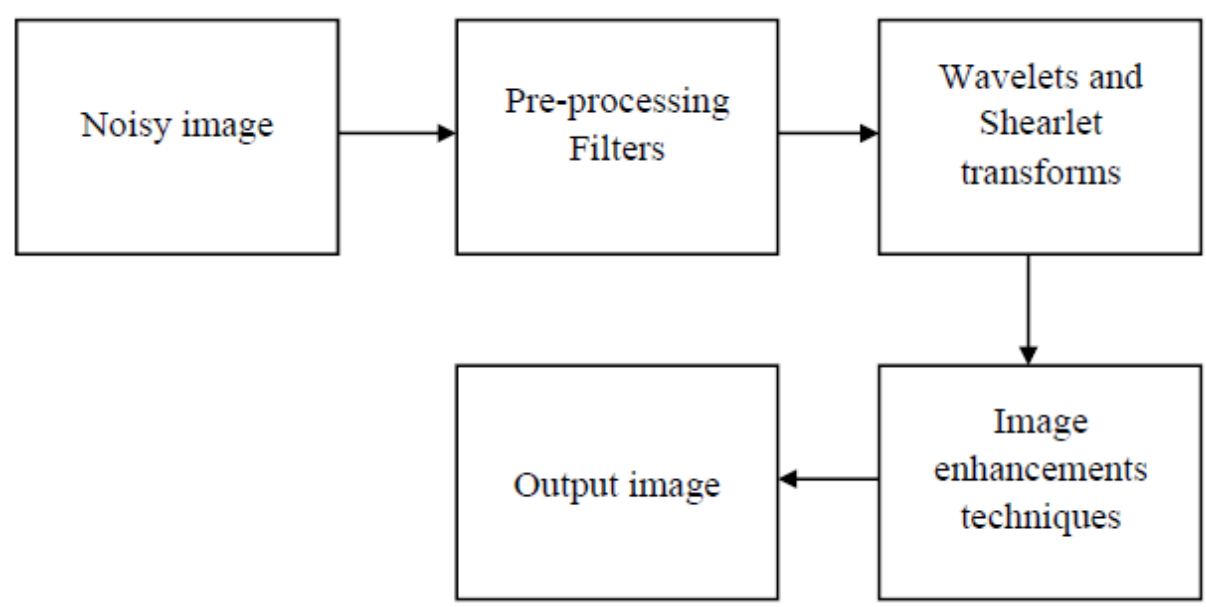

Figure 4:

As described in block diagram, the noisy image is preprocessed by using filters like linear or non-linear filters. Denoising can be done using different methods like linear or non-linear filters or techniques using algorithms. The linear filters are known as mean filters or low pass filters. The linear spatial filter calculates the average of pixels in the neighborhood of the filter mask. These filters sometimes are called averaging filters. The output is obtained by replacing the average of the gray levels in the neighborhood defined by the filter mask. This process reduces the sharp transitions in gray levels. The non-linear filters are minimum filters, maximum filters 
Recent Advances in Communication, Electronics \& Electrical Engineering

and median filters. Median filter reinstates the value of a pixel by the median of the gray levels in the neighborhood of that pixel. These median filters are popular in providing excellent noise reduction and effective in the presence of salt and pepper noise. Minimum filter replace the pixel value with minimum value in the corresponding neighborhood. Maximum filter replaces the pixel value with maximum value in the corresponding neighborhood. The filtered image is sent for further process using Wavelet and Shearlet transforms where noise is removed effectively and edge detection takes place. Wavelet and Shearlet have a common advantage on Multiresolution where image is processed in multidirection. Histogram, color image processing provides good results in image enhancement process. The whole process is simulated using MATLAB 2016b.

\section{Results and Discussions}

We have denoised the noise corrupted image using non-linear filters which is simulated in MATLAB tool and obtained the results. The non-linear filters are best among the filters in removing noise to the maximum extent. During the course, some amount of information is lost where image lost its quality, So that the image should be smoothened and sharpened in order to retrieve the original information. The below images represent the denoised results using nonlinear filters which are corrupted by noise like Gaussian, salt and pepper and speckle noise respectively

- The below image shows the denoised results of Gaussian noise.

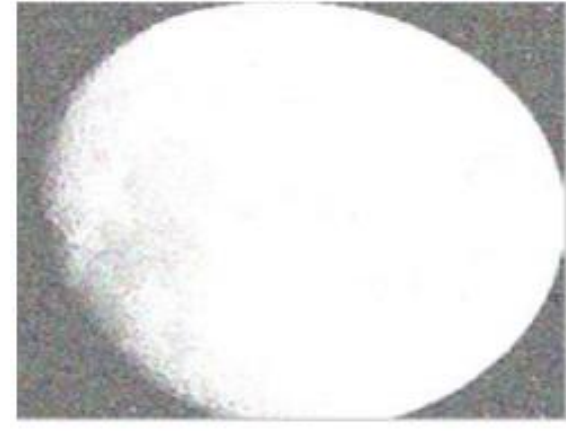

Figure 5: Noisy image

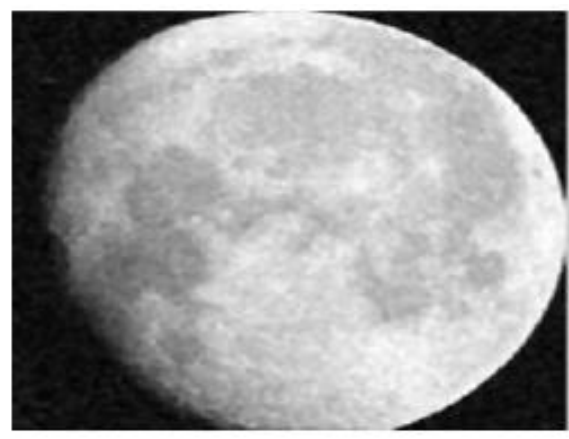

Figure 6: denoised image

- The below image shows the denoised results of salt and pepper noise

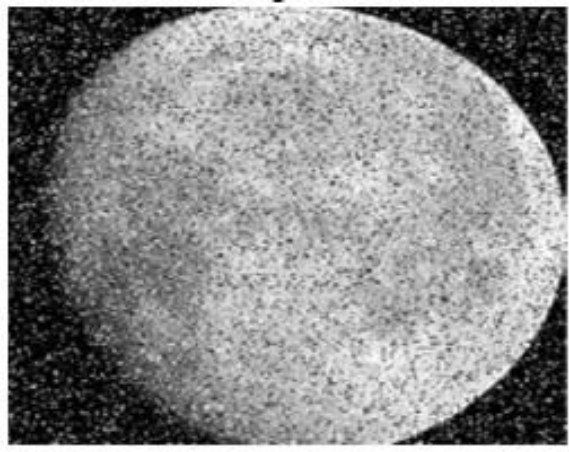

Figure 7: Noisy image

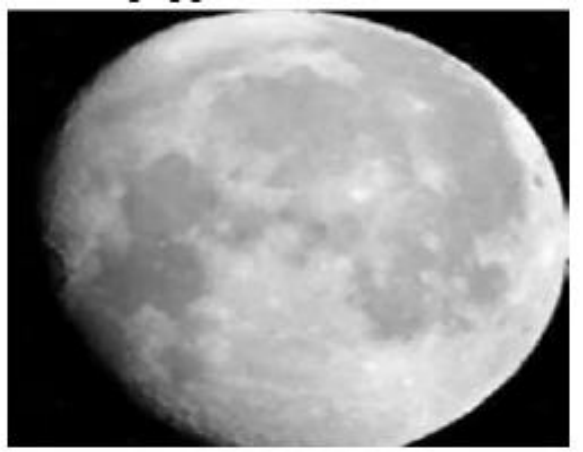

Figure 8: denoised image 
Recent Advances in Communication, Electronics \& Electrical Engineering

- The below image shows the denoised results of speckle noise

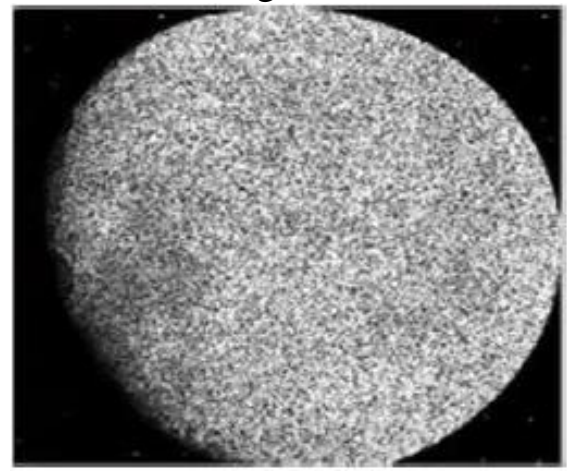

Figure 9: Noisy image

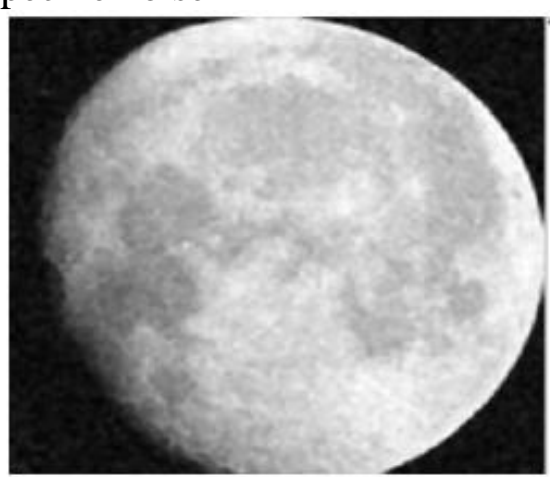

Figure 10: Denoised image

\section{Conclusions \& Recommendations}

As we seen from the results that the images had lost some quality during denoising using nonlinear filter. We use better techniques like Wavelet and Shearlet transforms together which is known for fetching the high value of PSNR where it is indirectly improving the quality of the image along with effective removal of noise and edge detection. These denoising techniques restore the information of the image and help in desired application field.

\section{References}

[1] Digital Image Processing (second edition)-by RAFAEL C.GONZALEZ and RICHARD E.WOODS.

[2] T.S.ANJU and N.R.NELWIN RAJ, "Shear-let transform based image de-noising using histogram thresholding." IEEE Trans. Communication Systems and Networks, vol.38, no.2, 2016, pp.162166.

[3] Malini.S and Moni.R.S, "Multi-resolution Image De-noising for Detection of Bridges in Satellite Images," IEEE Trans .on image processing, 2015, pp.457-461.

[4] S.Mallat and W.L.Hwang, "Singularly Detection and Processing with Wavelets." IEEE Trans. Information Theory, vol.38, no.2, March 1992, pp.617-643.

[5] J.L.Starck, E.J.Candes, and D.L.Donoho, "The curvelet transform for image de-noising" IEEE Trans .on image processing, vol.11,2002,pp.670-684.

[6] B.N.ARAVIND and K.V.SURESH, "An Improved Image De-noising Using Wavelet Transform" IEEE Trans.on image processing, vol.01,2015,pp.1-5.

[7] MUHAMMAD SAJID and Dr.KHURRAM KHURSHID, "Satellite Image Restoration Using RLS Adaptive Filter and Enhancement by Image Processing Techniques", IEEE Trans. on image processing,2015,pp.1-7.

*Corresponding author.

E-mail address: bharathkumar281095@gmail.com 